\title{
Design thinking based on reflective level in emotional design
}

\author{
Y i Chen ${ }^{1 *}$, Runchu Chen ${ }^{2}$ \\ ${ }^{1}$ College of M echanical and Electrical Engineering, Hohai U niversity, Changzhou, Jiangsu, 213022, China \\ ${ }^{2}$ College of M echanical and Electrical Engineering, Hohai U niversity, Changzhou, Jiangsu, 213022, China
}

\begin{abstract}
This article discusses the connotation and value of reflective level design. By analyzing some products of the four angles of reflective level including self-image expression, reflection of implication and understanding, narrative interpretation and metaphor, this article gives deep reflections of the reflective level product design. Finally, this article proposes a creative product design method based on emotional design, develops and enriches the current design methodology, and provides a new idea and possibility for cultural product design.
\end{abstract}

\section{Introduction}

With the improvement of industrial technology level, emotional expression gradually occupies an important position in the market. The term "emotion" comes from the field of psychology, and it is a physiological response of man to external things when they act on themselves, and is determined by needs and expectations ${ }^{[1]}$. For design, emotion is a means of transmission, the strategic significance of emotional design is whether the design can mobilize the user $\odot$ psychology, and produce the expected emotional response, so that the user can produce a sustained and benign experience. Therefore, what is the most important of emotional design is to improve the interactive experience and emotional experience of the use process.

Donald Norman ${ }^{[2]}$ divided people@ emotional experience of objects into three categories according to the level of brain activity: visceral level, behavioral level and reflective level. Among them, the design of the reflective level brings the feelings of users to the level of understanding. At this level, the emotional experience brought by goods and services comes from people@ high-level thinking activities ${ }^{[3]}$. Good reflective level design can produce emotional communication with users, and make consumers produce emotional dependence and even brand loyalty.

\section{Case study of emotional design products}

According to the level of reflection in the three levels of emotional design, the article carries out the analysis and discussion of products from four perspectives, including self-image expression, the implication and understanding, narrative interpretation, and metaphor.

\subsection{Case of self-image expression}

For users, the value of an object includes many additional hidden values. Consumers choose to buy a product to a large extent which is even more value-added. A product is a mirror, reflecting in the transmission of consumer identity, status, personality, preferences, values and even lifestyle. Today, electronic cameras and mobile phone cameras are becoming more and more advanced and developed. There is still a group of so-called "mechanical enthusiasts" pursuing pure and extreme mechanical film cameras. From a psychological point of view, "enthusiast" means a self-identity. The camera product at this time was not only a photographic tool, but also a collectible, a commemoration of an era, a symbol of pursuit and refinement. Figure 1.

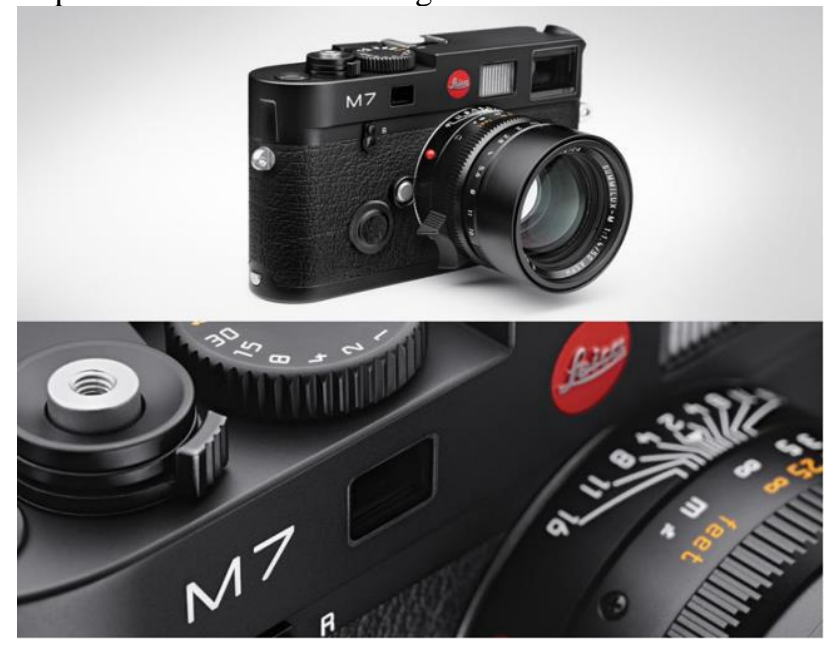

Figure 1. Leica M 7 Details 


\subsection{Case of reflection on the implication and understanding}

In the level of reflection, technology, the emphasis should be more on the process, that is, the value of the user experience layer. A good product can talk to people in the process of interacting with them. On the one hand, users can generate new cognition and new experience because of interesting interaction modes, on the other hand, they can gain culture through using products.

Today, calendar is more than just a date. The time and years mapped by the calendar have a certain carrying capacity, which carries human memories, culture and feelings. U sers flip through the cal endar every day, which is a kind of enjoyment and knowledge acquisition of its illustration design, text content, and historical stories.

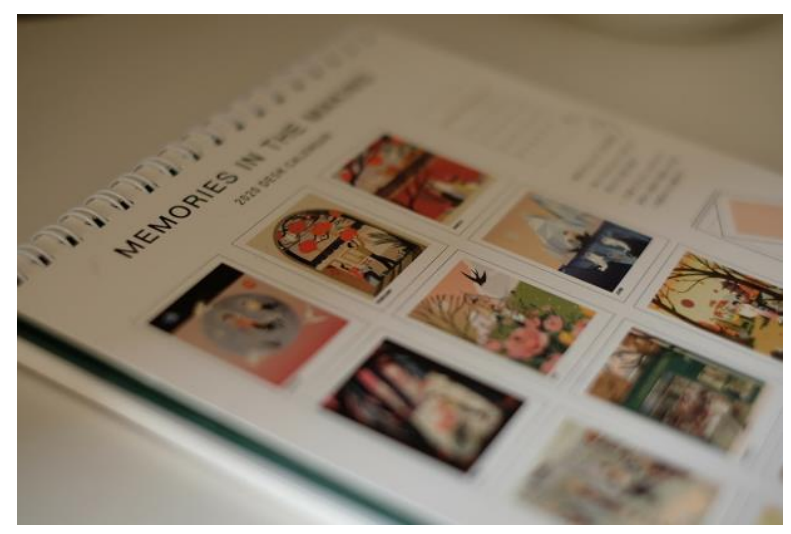

Figure 2. N etE ase N ews $\odot 2019$ ttitude C al endar

At present, the market capacity of cultural and creative products is huge. $M$ ore and more products begin to explore creative elements, play cross-border, new media, or even carry out brand-new design and packaging promotion based on the Internet. This makes users have a strong emotional experience, association and even re-creation. For example, museum design and cultural travel design, the essence of which is to allow users to simultaneously obtain the additional value of the culture, memory, and emotion behind the product when facing the product.

\subsection{Case of narrative interpretation}

Narrative used to be a word of contemporary literature, which belongs to the category of narratology. In terms of design art, the narrative performance of design works is based on the design of objects or images, and is more dependent on the user $($ association and imagination to supplement. Taking children $\odot$ education products as an example, the coding suit Kano Harry Potter Wand developed by $\mathrm{K}$ ano and $\mathrm{W}$ arner B ros Consumer Products has an emotional packaging design in terms of narrative interpretation. The film plot is combined to give the product legend and story. Figure 3.

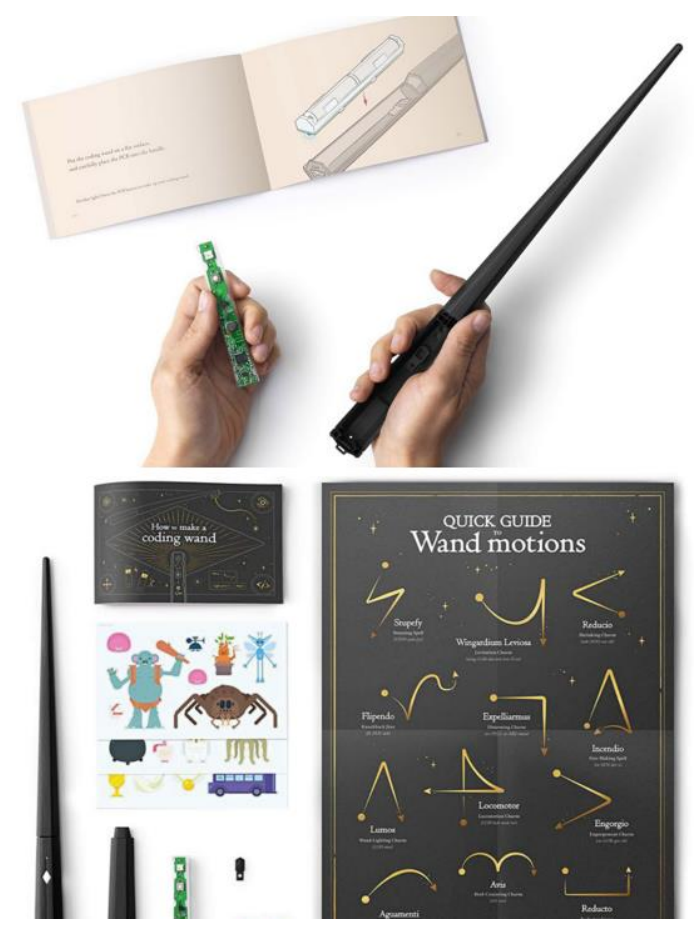

Figure 3. Canoe Harry Potter programming game wand set

For high-tech products, how to break the cognitive barriers of consumers, grasp their consumption psychology and stimulate consumption is very important, which requires product designers to give them legendary and storytelling in their appearance or use experience. The coding game product, which pays tribute to harry potter $\$$ magic wand, captures the group of movie and television fans, combining ordinary technology products with movies with many loyal fans, making users have a strong sense of participation and bringing in. What's more, they will even add their own understanding and association to deepen product dependence.

\subsection{Case of symbols and metaphors}

The symbol refers to the way designers use images or relevant elements to design as symbols and metaphors to make people experience special emotions. Some metaphorical expressions are obvious, but some need users to think and associate life to decipher its meaning.

In music player interface, designer uses a metaphorical design method, showing the current play song information in the form of vinyl records. The singing needle is placed on the record, and the record rotates at a constant speed and slowly represents that the current song is playing. When the singing needle leaves the record, the record stops spinning on behalf of the current song for playback pause. It not only embodies the efficient transmission of information function, but also brings users interesting interactive experience. M etaphor performance process often has stronger psychological cues and operational feedback, and is also the most worthy of study in the interactive design of metaphor design. 


\section{Emotional Design Strategies for Creative Products}

\subsection{The acquisition of empathy}

Sympathy acquisition refers to the user(s) ability to quickly generate a sense of identity and intimacy. For cultural and creative products, the target group is more young users, so in the product packaging publicity should pay attention to the form of expression, close to the young key words such as "fashion, fresh, interesting" design, bold cross-border and improvement.

\subsection{Access to a sense of participation}

Sociality is the basic attribute of human beings. And the sense of participation and belonging is indispensable in the process of meeting the high-level needs. The acquisition of a sense of participation requires the necessary, interesting interaction or social behavior of the person in the process of using. By dividing the characteristics of the target population, analyzing the user $\odot$ psychology and adding appropriate interaction in the product design, it can improve the user@ use experience to a certain extent.

\subsection{Acquisition of controllable sense}

$M$ artinE. P.Seligman ${ }^{[4]}$ points out that modern people@ sense of control over the outside world can bring positive emotions. In the design of the product, designers should grasp the psychological characteristics of the user to carry out the appropriate "blank space" that gives the user a part of the imagination and creation. As for the user-centered design, this design is more hel pful to leave loyal users, increase the user stickiness of the product brand.

\section{Conclusion}

Emotional design is a basic form of emotional communication between users and products. It provides a new perspective for product design and increases emotional communication between users and products ${ }^{[5]}$. In the theory of visceral level, behavioral level and reflective level proposed by Professor Donald Norman for affective design, the reflective level is in the high level design, which is dominant in the instinct layer and the behavior layer. Compared with the first two levels, it is consciously present and can arouse the user@ memory and thinking, interpret and understand the product from all aspects, therefore, it is also easy to change with the user $\odot$ consciousness ${ }^{[6]}$. Through the design case analysis based on the level of reflection, it can make the designer better grasp the user $\odot$ psychology and help design people-oriented, temperature-rich and human creative products.

\section{References}

1. Zhu Yuan. Research on Emotional Interaction Design in Wearable Smart Products [J]. Arts and Design (Theory), 2015,2 (12): 102-104.

2. Donald Norman. Emotional Design [M]. Beijing: CITIC Publishing Group, 2015.

3. Donald A. Norman. Design Psychology [M ]. CITIC Publishing House, 2003.

4. Xiao Dongmei. Happiness and its cultivation [D] .Hunan U niversity, 2012.

5. Huang Huiqin, Xie Yuwei.Research on bamboo lamps based on emotional design [J] .Packaging Engineering, 2019,40 (20): 164-168.

6. Zhang Kai.Thinking about the reflection level of traditional folk creation art in emotional design [J] .J ournal of Hubei University of Economics (Humanities and Social Sciences), 2015,12 (02): 19-21. 